\title{
Formation en radioprotection du personnel des entreprises intervenant en centrale nucléaire
}

\author{
J. CONSTANCIS et A. GAUTHIER $(*)$
}

(Manuscrit reçu le 4 février 1980)

\begin{abstract}
RÉSUMÉ
Depuis plus de 10 ans, les A.P.A.V.E. ont entrepris, à la demande de leurs adhérents, des actions de formation en radioprotection dans le prolongement de leurs activités de contrôle des sources de rayonnements ionisants en milieu industriel comme en milieu médical. C'est donc du fait de cette expérience que les A.P.A.V.E. ont été sollicitées pour assurer la formation en radioprotection du personnel des entreprises appelé à intervenir en centrale nucléaire. Depuis plus de 3 ans, près de 5000 personnes ont suivi ces cycles de formation. Le présent exposé tente donc de retracer cette démarche, dresse un premier bilan de cette action et livre quelques réflexions.
\end{abstract}

\section{SUMMARY}

For more than 10 years, in order to meet the wishes of their members, the A.P.A.V.E. associations have organised training courses in personnel radiation protection, as a consequence of their activities in the inspection of ionizing radiation sources in industrial or medical environments. Because of their experience, the A.P.A.V.E. associations were asked to provide for the training of the firm personnel likely to work in nuclear power stations, in the field of occupational radiation protection. For the last 3 years, nearly 5,000 people have attended these training sessions. The present report describes the approach, draws the first conclusions and state some considerations on this subject.

\section{INTRODUCTION}

Compte tenu des obligations de formation découlant des prescriptions du décret du 28 avril 1975 relatif à la protection des travailleurs contre les dangers des rayonnements ionisants dans les installations nucléaires de base,

(*) Centre technique et énergétique de l'Association lyonnaise des Propriétaires d'Appareils à vapeur et électriques (A.L.P.A.V.E.), 177, route de Sain-Bel, 69160 Tassinla-Demi-Lune.

RADIOPROTECTION, VOL. 15 - 0033/8451/1980/147/\$ 5.00/C) Bordas-Dunod. 
les A.P.A.V.E. ont été, très tôt, sollicitées pour assurer la formation en radioprotection du personnel des entreprises appelé à intervenir en centrale nucléaire. A ce jour, les A.P.A.V.E. dispensent cette formation sur les sites de Fessenheim, Bugey, Tricastin, Dampierre et Gravelines, sans compter les nombreux stages réalisés au sein même des entreprises ou aux sièges des A.P.A.V.E.

L'élaboration de cette formation est liée à une étroite collaboration avec l'exploitant des centrales nucléaires et à des échanges de vue toujours fructueux. Cette action, enfin, a pu être menée à bien grâce au rôle efficace des cadres de sécurité des collèges interentreprises implantés sur les différents sites. Pour réaliser ces actions, les A.P.A.V.E. ont utilisé une démarche particulière, à savoir : constitution d'un groupe préparatoire pour une première réflexion, analyse et définition d'un programme, conception de l'action et élaboration des méthodes et outils pédagogiques, organisation et animation de l'action.

\section{BUTS DE LA FORMATION}

Le but de cette formation est explicité par le décret du 28 avril 1975 qui précise dans son article 11 : «L’employeur doit préparer au travail sous rayonnements tout travailleur affecté dans la zone contrôlée, ou appelé à y travailler occasionnellement et l'informer par une notice appropriée :

- des risques de contamination ou d'irradiation auxquels son travail est susceptible de l'exposer;

- des précautions à prendre pour éviter ces risques;

- des méthodes de travail offrant les meilleures garanties de sécurité;

- des garanties que comportent pour lui les mesures physiques et les examens médicaux périodiques.

Cette formation et cette information devront être adaptées à la nature des travaux effectués et aux travailleurs auxquels elles s'adressent. "

En fonction de cette obligation réglementaire nous nous sommes fixés les objectifs suivants :

- donner à chaque travailleur les moyens de se comporter correctement face à un risque peu familier et méconnu, voire inquiétant, pouvant créer la peur ou des réactions incontrôlées;

- appréhender, dans la mesure du possible, les conséquences de la radioprotection vis-à-vis des méthodes habituelles de travail auxquelles le personnel était accoutumé, voire vis-à-vis de l'aspect économique des interventions en centrale nucléaire;

- donner aux personnes devant être qualifiées en radioprotection (telles que définies à l'article 9 du décret) les moyens d'accomplir leur tâche dans la limite des responsabilités qui leur sont assignées.

Pour atteindre les objectifs ainsi définis et répondre à l'esprit de la règlementation, les A.P.A.V.E. ont constitué un groupe préparatoire appelé à participer à toutes les étapes de la démarche. 


\section{3. ÉLABORATION DU PROGRAMME DE FORMATION}

Deux critères ont présidé à l'élaboration de ce programme :

- d'une part être adapté aux travailleurs D.A.T.R. $\left({ }^{1}\right)$ auxquels s'adresse cette formation. Ceci a nécessité la définition de deux niveaux de formation conformes à ceux proposés par E.D.F., l'un destiné aux "Chefs de travaux ", personnel qualifié en radioprotection, l'autre destiné aux exécutants intervenant sous la surveillance directe d'un chef de travaux;

- d'autre part aborder des notions qui, bien que relevant de l'information, constitueront des éléments nécessaires pour placer à un juste niveau les problèmes pratiques en offrant même la possibilité de réagir correctement devant des situations imprévues. Cette part d'information dispensée de façon objective constitue également un élément qui peut éviter les conflits risquant de naître lorsque l'information est donnée de façon tronquée ou partielle.

Par ailleurs, il importait de bâtir un programme qui prenne en compte les thèmes définis avec l'importance qui leur est attachée et selon une progression méthodique. Ce programme a donné lieu à l'élaboration de dossiers pédagogiques fixant d'une part le contenu de la formation et d'autre part les méthodes et outils dont disposent les instructeurs.

\section{4. ÉLABORATION DES MÉTHODES ET OUTILS PÉDAGOGIQUES}

Les méthodes retenues doivent permettre :

- de s'adapter correctement aux stagiaires selon leur niveau de connaissance et leur expérience éventuelle du nucléaire;

- de réaliser cette formation dans le strict respect du temps imparti;

- de simuler des situations pour analyser des gestes, des initiatives ou un comportement;

et ce, en garantissant dans toute la mesure du possible, l'assimilation et l'appropriation des notions essentielles qui conditionneront la juste réaction sur le terrain.

Les dossiers pédagogiques élaborés par le groupe préparatoire et utilisés par les instructeurs permettent le respect de ces critères et constituent, en outre, une sorte de spécification garantissant l'identité des stages entre eux. Ils définissent, également, un découpage des séances en fonction des durées fixées et la répartition des exposés entre les instructeurs. Les méthodes utilisées sont bâties sur les principes suivants :

- exposés courts, articulés autour de schémas et tableaux;

- utilisation des moyens audiovisuels;

- actions pratiques avec participation active des stagiaires.

(i) D.A.T.R., directement affectés aux travaux sous rayonnement. 
Les exposés constituent une part de démonstration à laquelle viennent s'intégrer des actions interrogatives stimulant la réflexion et permettant l'appropriation progressive des notions essentielles. Toutefois, cette méthode ne trouve sa pleine efficacité que dans la sollicitation permanente des stagiaires.

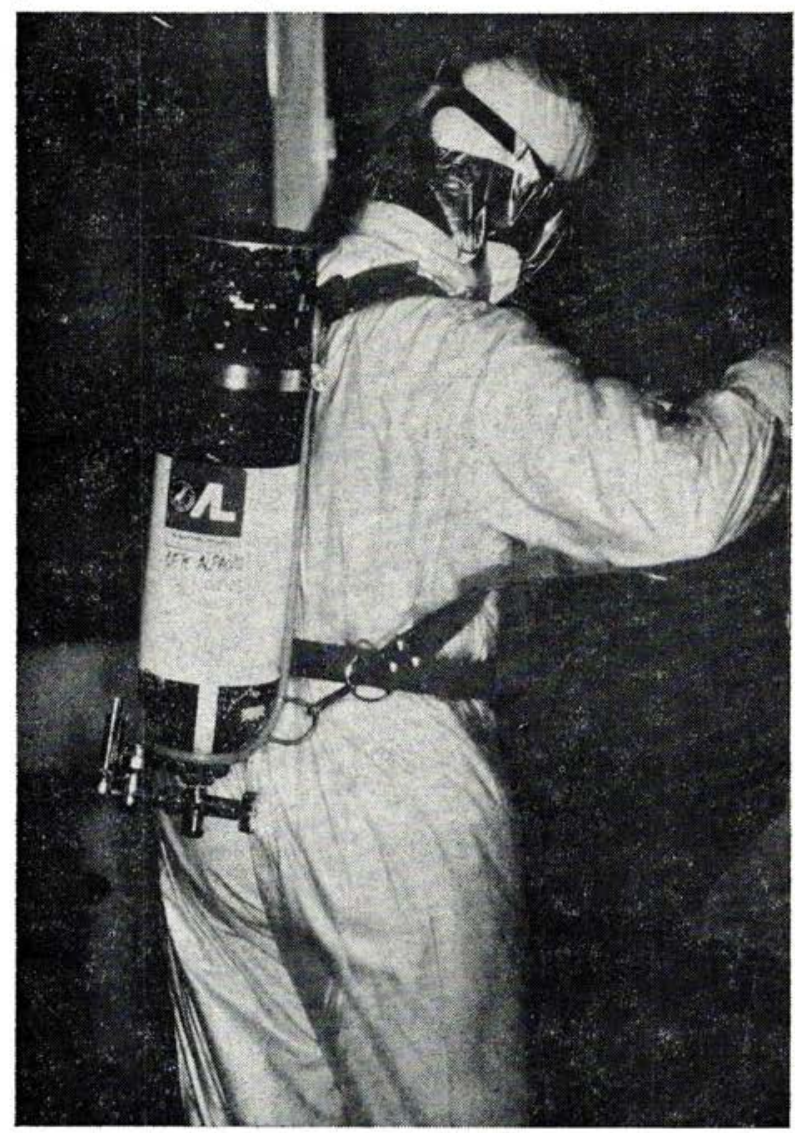

Contamination : entraînement à l'utilisation de tenues spéciales.

Les moyens audiovisuels sont volontairement limités sous peine d'isoler le stagiaire et de perdre sa participation. Toutes les présentations de films, diapositives ou tableaux sont suivies obligatoirement de commentaires sous forme d'échanges entre instructeurs et stagiaires. Les moyens utilisés sont donc parfaitement intégrés à l'action des instructeurs. En particulier, les exposés sont illustrés à l'aide de schémas composites bâtis au fur et à mesure du déroulement de l'exposé.

Les actions pratiques tiennent, bien sûr, une large place, même si celle-ci peut être jugée encore insuffisante. Les méthodes et moyens de mesure mis 
en œuvre, que les stagiaires abordent par groupes, sont similaires à ceux qu'ils utiliseront sur les sites. Les mesures, notamment, sont interprétées, discutées, analysées et les principes ou règles de protection à adopter sont mis chaque fois en évidence. Il en est de même pour l'entraînement à l'utilisation

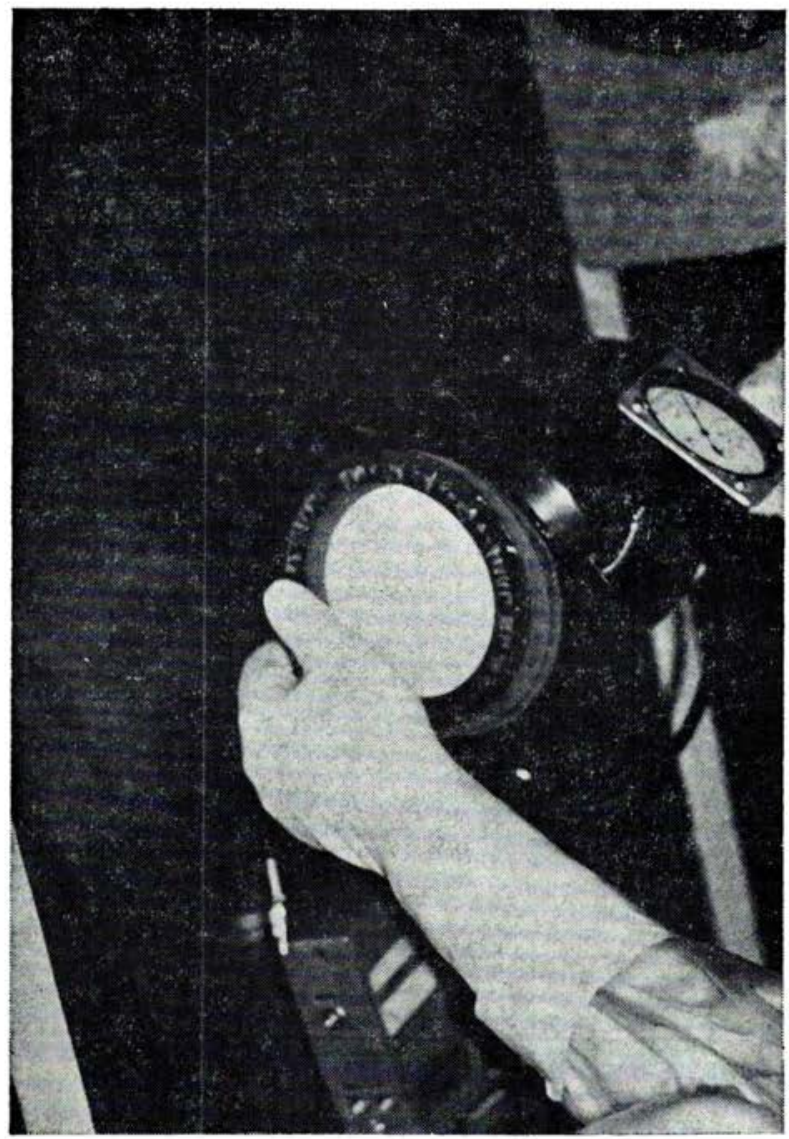

Contamination : prélèvement atmosphérique.

des moyens de protection individuelle où les principes sont particulièrement détaillés ce qui, à terme, constituera vraisemblablement un gain de temps sur le site et accroîtra la sécurité. En fin de stage, pour les chefs de travaux, l'exercice pratique appelé " Chantier École " simule une intervention et de ce fait favorise la synthèse des notions acquises tout au long du stage. Cet exercice constitue, sans doute, un des meilleurs éléments pour amener le stagiaire à acquérir les notions primordiales concernant l'évaluation des risques, la détermination des mesures de protection à mettre en œuvre pour recevoir

VOL. $15-\mathrm{N}^{\circ} 3$ 
la dose la plus faible possible. De plus, les stagiaires qui interviendront ensuite sur un site nucléaire comprendront plus facilement les raisons de certaines prescriptions et les mesures de sécurité qu'ils devront prendre seront acceptées et intégrées à leur intervention, sans que subsiste en eux une arrière pensée pouvant créer une sorte de méfiance, qui pourrait atténuer leurs moyens.

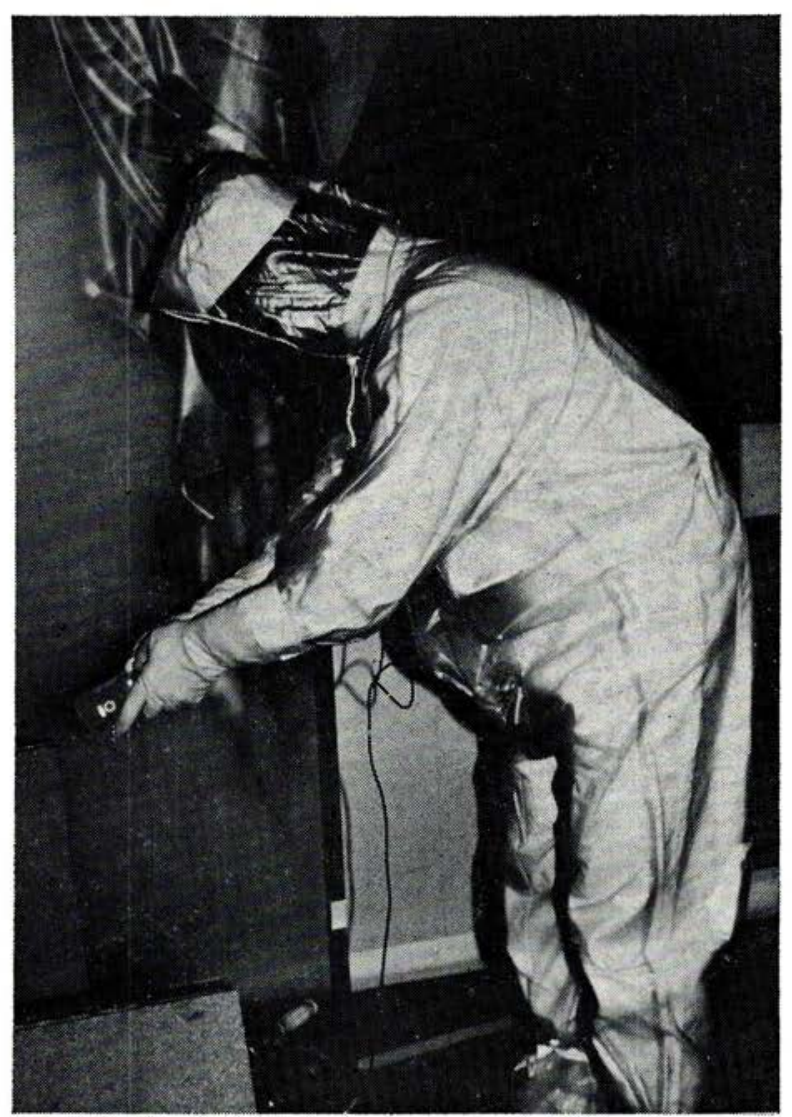

Irradiation : détection d'une source et mesure du débit de dose.

Pour parvenir à un résultat positif, toutes ces méthodes doivent :

- faire appel à un vocabulaire précis;

- permettre une approche progressive des difficultés;

- comporter la reprise régulière des notions déjà acquises (séances de rappel); 
- être simples pour que la compréhension soit possible par tous malgré l'hétérogénéité des stagiaires;

- donner la possibilité aux instructeurs de s'adapter en permanence au groupe.

Pour toutes les actions pratiques, les instructeurs jouent le rôle d'animateurs de groupe en contrôlant en permanence l'action de chaque stagiaire. Ce contrôle qui permet de déceler chez chacun les éventuelles difficultés et ce, au moment où elles surgissent, est un facteur de réussite de la formation.

\section{ANIMATION DE L'ACTION}

L'animation de l'action est, en partie, définie par les moyens pédagogiques adoptés. Elle doit répondre au but fixé, c'est-à-dire que l'information soit transmise, comprise, assimilée et donc utilisable lors d'une intervention. Ce but ne peut être atteint sans une équipe d'instructeurs assez large et homogène, sans parler bien sûr de leur compétence ou de leurs aptitudes pédagogiques. Les A.P.A.V.E. disposent actuellement d'une équipe d'environ 25 instructeurs diplômés en radioprotection et dont la plupart possèdent déjà une expérience confirmée de la formation.

Compte tenu de l'importance de l'impact que peut avoir cette formation tant sur le plan de la sécurité que sur le plan économique, les stages sont conduits et dirigés en permanence par deux instructeurs selon le principe de la co-animation, c'est-à-dire intervenant à tour de rôle ou simultanément pour les exercices pratiques. Cela permet de regrouper 14 à 15 stagiaires par session. De plus, en présence de deux instructeurs, la participation des stagiaires a plus de chance de se manifester de façon efficace. En effet, même si la formation revêt un caractère obligatoire, celle-ci doit être acceptée et non subie, et c'est, bien sûr, le rôle des instructeurs de veiller à ce qu'il en soit ainsi. D'autre part, les documents remis aux stagiaires ne doivent être utilissé que pour compléter leur formation ou reprendre des points qu'ils peuvent avoir oubliés, mais ils ne constituent pas l'élément essentiel de la formation.

De façon annexe, ces stages doivent mettre aussi en évidence les contraintes qu'imposent la radioprotection et ses conséquences économiques. C'est là un point très important souvent ignoré, mais qu'il n'est pas possible de ne pas évoquer. Dans ces conditions, les A.P.A.V.E. ont retenu, pour proposer aux entreprises, des stages de 4 ou 5 journées pour les chefs de travaux et de 2 ou 3 journées pour les exécutants et ce, à la demande des collèges de sécurité inter-entreprises et en accord avec les exploitants.

Compte tenu des prescriptions de la réglementation en vigueur et des responsabilités incombant à chaque employeur, il est du devoir de l'organisme qui se voit confier une telle formation, de s'assurer de façon précise de la qualité des connaissances acquises. En effet, l'organisme formateur doit jouer un véritable rôle d'assistance auprès des stagiaires et fournir à leur employeur un avis sur les aptitudes de son personnel face aux risques rencontrés en centrale nucléaire.

VOL. $15-\mathrm{N}^{\circ} 3$ 
Les instructeurs s'assurent donc de la qualité des connaissances acquises, d'abord au cours du stage tant dans les exercices théoriques que pratiques et, ensuite, à l'issue du stage où il est procédé à un contrôle des connaissances composé, en partie, de questions à choix multiples et, en partie, d'exercices simples représentatifs de cas concrets. Les réponses au questionnaire sont ensuite examinées de façon qualitative.

Ainsi, s'appuyant sur ces critères de jugement, les instructeurs formulent à l'employeur un avis sur l'aptitude du stagiaire à remplir la fonction proposée. Les avis formulés sont de trois types :

- avis favorable;

- avis réservé;

- avis défavorable.

\section{TABLEAU I}

NOMBRE DE STAGIAIRES

(bilan au 31 juillet 1979)

Chefs de travaux

Nombre de stagiaires :

$$
\left.\begin{array}{lll}
\text { - sur site }: & 1378 \\
\text { - hors site : } & 497
\end{array}\right\} 1875
$$

Avis favorable : 1308 soit $69,8 \%$

Avis réservé : $\quad 411 \quad 21,9 \%$

Avis défavorable : $\quad 99 \quad 5,3 \%$

Sans avis: $\quad 57 \quad 3 \%$

environ 625 journées de formation

Agents d'exécution

Nombre de stagiaires :

$$
\left.\begin{array}{lrr}
\text { - sur site } & : & 2162 \\
\text { - } & \text { hors site } & 862
\end{array}\right\} 3024
$$

Avis favorable : 2396 soit $79,2 \%$

Avis réservé : $\quad 418 \quad 13,8 \%$

Avis défavorable : $155 \quad 5,1 \%$

Sans avis : $\quad 55 \quad 1,9 \%$

environ 500 journées de formation

Au total : 4900 stagiaires, 1125 journées de formation

$-38 \%$ chefs de travaux

- $62 \%$ agents d'exécution 
Si les avis favorable et défavorable sont sans ambiguïté, l'avis réservé traduit le fait qu'il n'a pas été possible de se prononcer sur l'aptitude immédiate du stagiaire à la fonction proposée et qu'une formation complémentaire spécifique s'avère nécessaire. Bien entendu, les avis prononcés ne peuvent pas préjuger de la décision de l'exploitant soit quant à l'agrément du travailleur s'il s'agit d'un " chef de travaux ", soit quant à l'acceptation ou au maintien du travailleur s'il s'agit d'un « exécutant ».

Dans le cas des stagiaires ayant fait l'objet d'un avis réservé, il est généralement proposé des séances de rattrapage essentiellement consacrées à la critique du stage ayant conduit à l'avis réservé, à l'analyse des erreurs commises et à une révision des notions essentielles en faisant très largement appel aux exercices pratiques. Un nouveau contrôle des connaissances est alors proposé au stagiaire ne pouvant conduire qu'à l'un des deux avis : favorable ou défavorable.

\section{PREMIER BILAN DE LA FORMATION}

Le premier bilan de cette formation qui s'étend maintenant sur plus de 3 ans est présenté sur les tableaux I et II. Le tableau I fait le bilan global au 31 juillet 1979 du nombre de stagiaires. Il est à noter que $38 \%$ des stagiaires ont suivi le niveau " chef de travaux » tandis que $62 \%$ ont suivi le niveau " exécutants ». La mention sans avis traduit les stagiaires qui n'ont suivi que partiellement le stage ou qui ont refusé le contrôle des connaissances.

Le tableau II fait le bilan au 31 mai 1979 des entreprises concernées par la formation sur le seul site du Bugey.

TABLEAU II

Nombre D'ENTREPRISES : POUR LE SITE DU BUGEY

(bilan au 31 mai 1979)

\begin{tabular}{|c|c|c|}
\hline \multirow[b]{2}{*}{ Nombre de stagiaires } & \multicolumn{2}{|c|}{ Nombre d'entreprises ayant présenté } \\
\hline & Chefs de travaux & Agents d'exécution \\
\hline 1 à $5 \ldots \ldots \ldots \ldots \ldots \ldots \ldots \ldots \ldots \ldots \ldots$ & 41 & 31 \\
\hline 5 à $10 \ldots \ldots \ldots \ldots \ldots \ldots \ldots \ldots \ldots$ & 16 & 12 \\
\hline 10 à $20 \ldots \ldots \ldots \ldots \ldots \ldots \ldots \ldots \ldots \ldots$ & 2 & 9 \\
\hline 20 à $50 \ldots \ldots \ldots \ldots \ldots \ldots \ldots \ldots \ldots$ & 5 & 6 \\
\hline 50 et plus..................... & 2 & 3 \\
\hline Total.......... & \multicolumn{2}{|c|}{$\begin{array}{l}79 \text { entreprises } \\
477 \text { stagiaires chefs de travaux } \\
785 \text { stagiaires exécutants }\end{array}$} \\
\hline
\end{tabular}

voL. $15-\mathrm{N}^{\circ} 3$ 


\section{CONCLUSION}

Il est sans doute difficile de tirer des conclusions sur une action qui est loin d'être à son terme. Juger, à l'heure actuelle, de l'efficacité de cette formation et du bien fondé des choix retenus est chose malaisée puisqu'un très grand nombre de personnes formées n'ont pas encore eu l'occasion de mettre en pratique les connaissances acquises, sinon de façon très ponctuelle et dans des situations ne présentant pas de risques majeurs. Pourtant, seule cette expérience pourra permettre un jugement et confirmer notre démarche. Il faut pourtant noter l'immense effort qu'il a fallu fournir pour atteindre et maintenir la qualité de cette formation et ce, malgré de nombreux obstacles, qu'il s'agisse de l'extrême hétérogénéité des niveaux des stagiaires rencontrés ou, quelquefois, des véritables cas de conscience imposés aux instructeurs lorsque l'avis qu'ils ont à formuler risque de conditionner l'avenir professionnel du travailleur.

Malgré le nombre important de personnes formées à ce jour, nous avons conscience que ces actions ne constituent qu'un premier pas vers la maîtrise du savoir faire que, seule, la pratique régulière, complétée par des séances d'informations, pourra permettre. Alors ces actions de formation apporteront à terme une contribution essentielle à la limitation de la radioexposition professionnelle, problème qui va prendre progressivement une importance considérable avec la mise en service des 40 à 50 tranches nucléaires engagées par E.D.F. 